\title{
Bronchial Fistula, CTCAE
}

National Cancer Institute

\section{Source}

National Cancer Institute. Bronchial Fistula, CT CAE. NCI Thesaurus. Code C143338.

A disorder characterized by an abnormal communication between the bronchus and another organ or anatomic site. 